\title{
RDUS
}

Revue de DROIT

UNIVERSITÉ DE SHERBROOKE

Titre : $\quad$ PERSONNES VULNÉRABLES ET CITOYENNETÉ : ÉVOLUTION ET PERSPECTIVES AUTOUR DE L'ACCESSIBILITÉ AU PROCESSUS DE VOTE

Auteur(s): $\quad$ Virginie MESGUICH

Revue : $\quad$ RDUS, 2009-2010, volume 40, numéro 1-2

Pages: $\quad 199-228$

ISSN : $\quad 0317-9656$

Éditeur : $\quad$ Université de Sherbrooke. Faculté de droit.

URI : $\quad$ http://hdl.handle.net/11143/10388

DOI : $\quad$ https://doi.org/10.17118/11143/10388 
Page vide laissée intentionnellement. 
ARTICLE

\section{PERSONNES VULNÉRABLES ET CITOYENNETÉ : ÉVOLUTION ET PERSPECTIVES AUTOUR DE L'ACCESSIBILITÉ AU PROCESSUS DE VOTE}

Les personnes handicapées et celles souffrant de maladie mentale font encore aujourd'hui l'objet d'une certaine stigmatisation qui limite leur accès à l'emploi, à l'éducation et même à la citoyenneté. Néanmoins, depuis la Loi électorale canadienne de 1970, qui refusait, le droit de vote aux élections fédérales $\grave{a}$ " toute personne restreinte dans sa liberté de mouvement ou privée de la gestion de ses biens pour cause de maladie mentale ", il faut admettre que de nombreux efforts, d'ordre législatif notamment, ont été réalisés. Ce texte a donc pour objectif de présenter l'évolution de l'accessibilité au processus de vote pour ces personnes dites vulnérables. Nous avons opté pour une présentation en deux temps. D'abord, le cadre international qui se justifie par le fait que le droit international joue un rôle de première importance dans la reconnaissance des droits de la personne dans le monde. Ensuite, le cadre national grâce auquel nous avons souhaité mettre en lumière les évolutions au Canada et évoquer les perspectives d'avenir.

Today, persons with disabilities, including those suffering from mental illness, remain subject to certain forms of stigmatization which limit their access to employment, to education and even to citizenship. Since the Canada Elections Act of 1970, which prohibited the right to vote in federal elections for "every person who is restrained of his liberty of movement or deprived of the management of his property by reason of mental disease", it must be acknowledged that numerous efforts, notably by the legislative branch to counter certain effects of this Act, have been put in place. The goal of this text is to describe the evolution of voting rights of those characterized as "vulnerable". This paper is divided into two parts, the first of which examines the international context, since international law plays a role of primary importance in the recognition of human rights worldwide. In the second part, the focus is on the national framework in order to emphasize the evolution of law in Canada and to suggest future developments.

Doctorante en droit, Centre de recherche en droit public (CRDP) de l'Université de Montréal. L'auteure remercie Emmanuelle Bernheim et Christine Vézina de lui avoir proposé de participer à leur colloque intitulé " Risques et populations marginalisées : une perspective de santé ", organisé dans le cadre du 77 e Congrès annuel de l'ACFAS, le 13 mai 2009. S'il est vrai que le thème n'était pas a priori en rapport direct avec la recherche doctorale de l'auteure, la discussion, présentée ici, ne manquait pas d'intérêt. Il s'agissait de réfléchir sur le thème général de la protection des droits fondamentaux dans la perspective du handicap et tenter de sensibiliser le lecteur à cette problématique. L'auteure tient également à remercier Arnaud Decroix, chercheur postdoctoral au CRDP et Emmanuelle Bernheim pour leurs précieux conseils. Les propos tenus dans cet article n'engagent que leur auteure. 


\section{SOMMAIRE}

I. Le cadre international ................................... 204

A) L'énoncé des grands principes de droit .............. 205

B) Les difficultés d'application........................... 215

II. Le cadre national .............................................. 219

A) Les changements apportés par la décision de la Cour fédérale du Canada ......................... 219

B) Les projets de réforme législative et les perspectives d'avenir ............................. 222

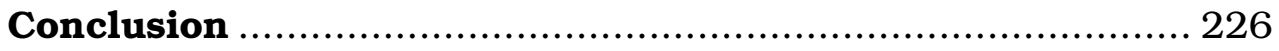


(2009-10) 40 R.D.U.S. évolution et perspectives
autour de l'accessibilité au processus de vote

"Les luttes pour la reconnaissance dans l'État de droit démocratique ont seulement une force de légitimation dans la mesure où tous les groupes peuvent avoir accès à l'espace public politique, peuvent y faire écouter leur voix, peuvent y articuler leurs besoins et qu'en outre personne n'est marginalisé ou exclu ".

Jürgen Habermas ${ }^{1}$

Jusque dans les années 1980, les personnes atteintes de maladie mentale, de déficience intellectuelle, les détenus et les personnes sans domicile fixe ${ }^{2}$ ne disposaient pas du droit de vote au Canada. La Loi électorale du Canada de 1970 refusait précisément le droit de vote aux élections fédérales à " toute personne restreinte dans sa liberté de mouvement ou privée de la gestion de ses biens pour cause de maladie mentale "3. Les raisons invoquées, à l'époque, pour justifier une telle interdiction reposaient sur une approche paternaliste. En effet, la tendance était bien plus orientée vers la protection des personnes vulnérables que vers leur participation pleine et entière à la société pour la simple raison qu'elles en étaient jugées incapables. Par ailleurs, un rapport direct était établi entre l'état de santé (physique ou mental) d'un individu et sa capacité à voter. Les personnes souffrant de troubles mentaux ou d'un handicap ont ainsi dû franchir de nombreux obstacles pour accéder aux conditions matérielles leur permettant de voter. Aujourd'hui encore nous observons un clivage entre l'état de santé et la

1. Jürgen Habermas, Textes et contexte : essai de reconnaissance théorique, Paris, Éditions du Cerf, 1994 tel que cité par Maria de los Angeles Yanuzzi, "Marginalisation sociale et perte des droits fondamentaux " dans Michel Coutu, Pierre Bosset, Caroline Gendreau, D. Villeneuve, dir., Droits fondamentaux et citoyenneté, une citoyenneté fragmentée, limitée ou illusoire?, Montréal, Thémis, 2005, à la p. 540.

2. Cette liste n'est pas exhaustive et le présent texte met l'accent sur les personnes atteintes d'un handicap physique ou mental et celles souffrant de maladie mentale.

3. Loi électorale du Canada, S.R.C. 1970, c. 14, art. 14 (4) [Loi électorale de 1970]. 
Personnes vulnérables et citoyenneté : évolution et perspectives (2009-10) 40 R.D.U.S. autour de l'accessibilité au processus de vote

perception d'une personne comme un électeur potentiel. Il est bien clair pourtant que l'interdiction de voter revient à isoler l'individu de la société, à lui retirer son droit d'expression, de participation à la vie sociale et démocratique.

Partant de la Loi électorale de 1970, nous avons souhaité comprendre l'évolution du droit au regard de ces populations que sont les personnes handicapées et celles souffrant de maladie mentale. Précisons immédiatement qu'il existe une distinction entre les personnes souffrant de troubles mentaux (paranoïa, schizophrénie, troubles bipolaires, dépression, etc.) et celles atteintes d'une déficience intellectuelle ${ }^{4}$. Pour cette seconde catégorie d'individus, il s'agit d'un état et non d'une maladie comme c'est le cas pour les personnes souffrant de problème de santé mentale. Il n'en demeure pas moins que les personnes concernées par l'une ou l'autre de ces situations méritent, tout autant, le bénéfice d'une accessibilité réelle au vote, sur la base notamment de la Charte canadienne des droits et libertés en respect de l'article $15(1)^{5}$. Notons que la Déclaration des droits $d u$ déficient mental de l'Assemblée générale des Nations Unies de $1971^{6}$ utilisait l'expression "handicap mental " non seulement pour les personnes atteintes d'une déficience, mais aussi pour celles souffrant de maladies mentales graves. À l'époque, plusieurs experts prétendaient que, dans un certain nombre de cas, une

4. Concernant le statut particulier des personnes présentant une déficience intellectuelle, lire l'article de Jocelyn Lecomte, "L'impact juridique réel et potentiel du droit international pour les personnes présentant une déficience intellectuelle au Québec ", École du Barreau du Québec, Justice, société et personnes vulnérables, Collection de droit, vol. hors série, Cowansville, Yvon Blais, 2008-2009.

5. Charte canadienne des droits et libertés, partie I de la Loi constitutionnelle de 1982, constituant l'annexe B de la Loi de 1982 sur le Canada (R.-U.), 1982, c. 11, art. 15 (1) [Charte canadienne] : "La loi ne fait acception de personne et s'applique également à tous, et tous ont droit à la même protection et au même bénéfice de la loi, indépendamment de toute discrimination, notamment des discriminations fondées sur (...) les déficiences mentales ou physiques ".

6. Déclaration des droits du déficient mental, Rés. AG 2856 (XXVI), Doc. off. AG NU, 26e sess., supp. n²9, doc. NU A/8429 (1971) 99 (2 août 2009), en ligne : <http://dcalin.fr/internat/droits_deficient_mental.html>. 
personne, en raison de sa maladie mentale, pouvait manquer de capacité juridique (c'est-à-dire sa capacité à exercer ses droits) et qu'en conséquence, certains de ses droits pouvaient être limités, ou même niés, afin de protéger ses intérêts grâce à des procédures judiciaires appropriées ${ }^{7}$. À partir de 1991, les principes onusiens pour la protection des personnes atteintes de maladies mentales et pour l'amélioration des soins de santé mentale 8 ont offert des garanties légales de protection à la fois aux personnes atteintes de maladies mentales et à celles ayant une déficience intellectuelle ${ }^{9}$. Les personnes déficientes intellectuelles étaient alors considérées comme ayant la capacité juridique d'exercer tous les droits de leurs concitoyens même si quelques-unes d'entre elles avaient des besoins spéciaux nécessitant une assistance particulière. Il paraissait, de fait, injuste de distinguer les personnes requérant des besoins particuliers et de restreindre leurs droits fondamentaux ${ }^{10}$.

7. Voir Klaus Lachwitz et Nancy Breitenbach, Droits de l'Homme et Déficience Intellectuelle, Londres, Inclusion International, 2002.

8. Principes pour la protection des personnes atteintes de maladies mentales et pour l'amélioration des soins de santé mentale, Rés. AG 46/119, Doc. off. AG NU, supp. no 49, Doc. NU A/46/49 (1991) (12 août 2009), en ligne :

<http://www.icrpd.net/ratification/documents/fr/Extras/Principles\%20 for\%20protection $\% 20$ of $\% 20$ persons $\% 20$ with $\% 20$ mental\%20illness.pdf>.

9. Ibid. Selon le paragraphe 6 du Principe 1 intitulé Libertés fondamentales et droits de base : "Toute décision selon laquelle, en raison de sa maladie mentale, une personne n'a pas la capacité juridique et toute décision selon laquelle en conséquence de cette incapacité, un représentant personnel sera nommé, ne sera prise qu'après que la cause aura été entendue équitablement par un tribunal indépendant et impartial institué par la législation nationale ". Dans le rapport rendu par Lachwitz et Breitenbach, supra note 7 à la p. 12, il est souligné que "Toutes les personnes handicapées mentales sont des citoyens de leur pays et ont le même droit que leurs concitoyens à la considération, au respect et à la protection par la loi (...) Une déficience intellectuelle ne justifiera en soi aucune forme de discrimination ".

10. Aujourd'hui, les Nations Unies considèrent que "l'incapacité peut consister en des déficiences d'ordre physique, intellectuel ou sensoriel, ou tenir à un état pathologique ou à une maladie mentale; elle peut être temporaire ou permanente " dans Règles pour l'égalisation des chances des handicapés, Rés. AG 48/96, Doc. off. AG NU, 48 e sess., Doc. NU (1993), art. 17, en ligne : <http://www.unhchr.ch/Huridocda/Huridoca. 
Personnes vulnérables et citoyenneté : évolution et perspectives (2009-10) 40 R.D.U.S. autour de l'accessibilité au processus de vote

Au-delà de l'importante question de la participation de tous, y compris des personnes souffrant d'un handicap ou de troubles mentaux, aux décisions et aux orientations d'une société, ce texte a également pour objectif de souligner la reconnaissance juridique des droits de ces personnes - dont l'accès à la citoyenneté - sur le plan international (I), puis sur le plan national, c'est-à-dire au Canada (II).

\section{Le cadre international}

Depuis 1993, date qui marque l'accessibilité universelle du vote au Canada, plusieurs pays ont reconnu le droit des personnes handicapées à l'intégration et à l'égalité dans la société dans laquelle ils vivent. L'existence d'un droit international de la santé a favorisé le développement de normes et de principes tendant à assurer le respect des droits fondamentaux, particulièrement pour les personnes vulnérables (A). Les Nations Unies ont notamment élaboré des Règles pour l'égalisation des chances des handicapés ${ }^{11}$ qui, bien que n'ayant pas de caractère contraignant, peuvent inspirer les pouvoirs publics dans la détermination de leurs politiques et leurs interventions donnant aux personnes handicapées les mêmes droits et les mêmes obligations qu'aux autres citoyens. Il n'empêche que l'application de ces règles et autres principes n'est pas toujours évidente et demande bien souvent des efforts de la part des États parties (B).

nsf/TestFrame/802558b7b5f9dc608025670c003c8adf?Opendocument.> [Règles pour l'égalisation des chances]. Notre exposé sera sensiblement différent selon que l'on s'intéressera à l'une ou l'autre de ces catégories d'individus.

11. Ibid. Voir aussi Canada, Ministère Ressources humaines et Développement des compétences du gouvernement du Canada, L'évaluation des politiques et des programmes destinés aux personnes handicapées: études - bilan - janvier 1999, en ligne: Ressources humaines et Développement des compétences Canada <http://www.rhdcc.gc.ca/fra/sm/ps/edd/rapports/1999000363/page03.shtml>. 
Personnes vulnérables et citoyenneté :
(2009-10) 40 R.D.U.S.
autour de l'accessibilité au processus de vote

\section{A) L'énoncé des grands principes de droit}

Le droit international joue un rôle de première importance dans l'essor des droits de la personne dans le monde depuis de nombreuses années ${ }^{12}$. Plusieurs normes internationales invitent les États signataires à prendre des mesures favorisant le plein épanouissement des droits et libertés des personnes, dans leur ensemble. Ce qui signifie, en outre, que les États doivent éviter d'entraver l'exercice de ces mêmes droits et libertés. Certaines de ces normes ont un caractère contraignant (les conventions et le droit coutumier international dès lors qu'ils ont été ratifiés), d'autres non-contraignant (avis d'experts ou d'organisations non gouvernementales). Le caractère contraignant est important en ce qu'il crée, en droit, une obligation pour l'État. La violation d'une telle norme soumet l'État en cause à un risque de sanction, de condamnation et à une réparation éventuelle. Toutefois, en fait, la situation n'est pas aussi radicale. Pour que la norme internationale ait force de loi, chaque État signataire doit légiférer. La situation du Canada, à cet égard, est particulière en raison du principe de partage de compétences entre le provincial et le fédéral, sur lequel nous reviendrons.

Sous la houlette du Haut-Commissariat aux droits de l'homme des Nations Unies, les professeurs Gerard Quinn et Theresia Degener, tous deux spécialisés dans le droit des personnes handicapées, ont produit un rapport, en 2002, intitulé Droits de l'homme et invalidité. L'utilisation actuelle et l'usage potentiel des instruments des Nations Unies relatifs aux droits de

12. Lire en ce sens la contribution de Caroline Gendreau, "Nouvelles normes internationales et droits fondamentaux : un problème d'effectivité du droit dans la création du droit " dans Michel Coutu, Pierre Bosset, Caroline Gendreau, Daniel Villeneuve, dir., Droits fondamentaux et citoyenneté, une citoyenneté fragmentée, limitée, illusoire?, Montréal, Thémis, 2005, 483. Gendreau met l'accent sur le droit créé sous l'égide de l'Organisation des Nations Unies relativement à la définition des droits fondamentaux en fonction de catégories particulières d'êtres humains (déficients mentaux, personnes handicapées, malades mentaux et personnes âgées). 
l'homme dans la perspective de l'invalidité13. Cette étude, de plus de trois cents pages, complète et documentée, a favorisé le débat sur les droits des personnes handicapées et a proposé des solutions pour " maximiser le potentiel des instruments des droits de l'homme des Nations Unies dans la perspective de l'invalidité ". Nous y ferons référence à plusieurs reprises.

$\mathrm{Au}$ point de départ des principaux instruments internationaux de protection des droits fondamentaux, se trouvent la Déclaration universelle des droits de l'homme ${ }^{14}$ (1948) et les Pactes internationaux relatifs aux droits civils et politiques ${ }^{15}$, d'une part, et aux droits économiques, sociaux et culturels ${ }^{16}$ (1966), d'autre part. Ils constituent la Charte internationale des droits de l'homme. Le Comité des droits de l'homme assure l'application du Pacte des droits civils et politiques dans les différents pays grâce à l'examen des rapports périodiques présentés par les États parties. Ce Pacte consacre des droits politiques classiques dont le droit à la liberté de pensée (art. 18), le droit à la liberté d'opinion (art. 19), le droit de prendre part à la direction des affaires publiques (art. 25), le droit à l'égalité (art. 2, 3 et 26), etc.

L'article 25 nous intéresse au premier chef puisqu'il énonce ce qui suit :

13. Gerard Quinn et Theresia Degener, Droits de l'homme et invalidité. L'utilisation actuelle et l'usage potentiel des instruments des Nations Unies relatifs aux droits de l'homme dans la perspective de l'invalidité, New York et Genève, Nations Unies, 2002, en ligne: Haut-Commissariat des Nations Unies aux droits de l'homme <http:// www2.ohchr.org/french/about/publications/docs/disability.pdf>.

14. Déclaration universelle des droits de l'homme, Rés. AG 217(III), Doc. off. AG NU, 3e sess., Doc. NU A/810 (1948) 71 (19 août 2009), en ligne: Nations Unies <http://www.un.org/fr/documents/udhr/>.

15. Pacte international relatif aux droits civils et politiques, 19 décembre 1966, 999 R.T.N.U. 171 (19 août 2009), en ligne : Haut Commissariat des Nations Unies aux droits de l'homme <http://www2. ohchr.org/French/law/ccpr.htm>.

16. Pacte relatif aux droits économiques, sociaux et culturels, 16 décembre 1966, 943 R.T.N.U. 13, en ligne : Haut-Commissariat des Nations Unies aux droits de l'homme <http://www2.ohchr.org/ french/law/cescr.htm>. 
Personnes vulnérables et citoyenneté :
(2009-10) 40 R.D.U.S.
autour de l'accessibilité au processus de vote

Tout citoyen a le droit et la possibilité, sans aucune des discriminations visées à l'article 2 et sans restrictions déraisonnables :

a) De prendre part à la direction des affaires publiques, soit directement, soit par l'intermédiaire de représentants librement choisis;

b) De voter et d'être élu, au cours d'élections périodiques, honnêtes, au suffrage universel et égal et au scrutin secret, assurant l'expression libre de la volonté des électeurs;

c) D'accéder, dans des conditions générales d'égalité, aux fonctions publiques de son pays.

On note, dès 1966, une véritable volonté d'intégrer tous les citoyens au processus démocratique et même de leur permettre "d'accéder aux fonctions publiques de [leur] pays ". Pour Gerard Quinn, l'article 25 peut s’interpréter " (...) de manière à assurer que tous puissent prendre part aux obligations civiques associées à la citoyenneté, le droit d'être juré d'assises étant un bon exemple "17. Ainsi les principes de non-discrimination fondée sur l'invalidité et le droit à l'égalité marquent de leur empreinte le Pacte des droits civils et politiques. Ils sont, certainement, parmi les principes les plus anciens et les plus fondamentaux des droits de l'homme. Les Règles d'égalisation des chances de 1993 y font expressément référence en disposant que :

25. Le principe de l'égalité de droits signifie que les besoins de tous ont une importance égale, que c'est en fonction de ces besoins que les sociétés doivent être planifiées et que toutes les ressources doivent être employées de façon à garantir à chacun des possibilités de participation dans l'égalité.

26. Les handicapés font partie de la société et ont le droit de rester dans leur collectivité d'origine. Ils doivent recevoir l'assistance dont ils ont besoin dans le cadre des structures ordinaires d'enseignement, de santé, d'emploi et de services sociaux ${ }^{18}$.

17. Supra note 13 à la p. 69.

18. Supra note 10. 
Personnes vulnérables et citoyenneté : évolution et perspectives (2009-10) 40 R.D.U.S. autour de l'accessibilité au processus de vote

L'Observation générale No25 de 1996 du Comité des droits de l'homme nuance ce propos en soulignant que les conditions s'appliquant à l'exercice des droits protégés par l'article 25 devraient être fondées sur des critères objectifs et raisonnables. Ainsi, l'Observation précise qu'il puisse être justifié de refuser le droit de vote ou celui d'occuper une fonction publique à une personne dont l'incapacité mentale est établie (art. 4)19. En conséquence, on ne peut ici refuser ou restreindre légitimement le droit de vote à une personne souffrant d'une invalidité physique ou intellectuelle, mais on le justifie dans l'hypothèse d'une incapacité mentale établie. Il n'est pas précisé, dans le texte, ce que le Comité entend par "incapacité mentale établie " et l'on ne peut s'empêcher de remarquer que cela constitue une limite au droit à l'égalité. Ce bémol crée, selon nous, une distinction qui n'a pas lieu d'être. Il ne peut y avoir deux poids deux mesures dans l'exercice du droit de vote. Une personne dont les facultés quelles qu'elles soient sont altérées peut toujours bénéficier de l'aide d'un préposé au vote ou d'une personne dont elle dépend. Cela pose cependant la question de l'autonomie et de la confidentialité du vote. Quelles sont les limites à ne pas franchir pour que le vote soit validé? Quels sont les risques de manipulation?

Bien que l'Observation ne donne pas de réponse stricto sensu à ces questions, elle précise tout de même au paragraphe 11 : " Les États doivent prendre des mesures efficaces pour faire en sorte que toutes les personnes qui remplissent les conditions pour être électeurs aient la possibilité d'exercer ce droit (...) "20.

Les principes de non-discrimination et d'égalité des droits sont donc à la base du Pacte des droits civils et politiques. On les retrouve également dans le Pacte des droits économiques, sociaux et culturels. En effet, aux termes de l'article 2, paragraphe 2 :

Les États parties au présent Pacte s'engagent à garantir que les droits qui y sont énoncés seront exercés sans discrimination aucune fondée sur la race, la couleur, le

19. Supra note 13 à la p. 75.

20. Ibid. à la p. 76 . 
Personnes vulnérables et citoyenneté :
(2009-10) 40 R.D.U.S.
autour de l'accessibilité au processus de vote

sexe, la langue, la religion, l'opinion politique ou toute autre opinion, l'origine nationale ou sociale, la fortune, la naissance ou toute autre situation ${ }^{21}$.

Remarquons la précision qui est ajoutée à la fin du paragraphe "ou de toute autre situation " qui offre tout un éventail de possibilités et laisse supposer que toute personne atteinte d'un handicap, ou d'une invalidité, n'a pas à souffrir de discrimination en raison de sa condition. À ce titre, donc, les États doivent élaborer et appliquer une législation antidiscriminatoire complète dans le sens de l'article $16 \mathrm{du}$ Pacte des droits économiques, sociaux et culturels ${ }^{22}$.

En conclusion, "[1]es principes de non-discrimination et du droit des personnes handicapées de jouir, sur un pied d'égalité avec les autres citoyens, de tous les droits de l'homme sont donc le souci majeur qui inspire les réformes, attendues depuis longtemps, sur la façon de considérer l'invalidité et les personnes handicapées partout dans le monde ${ }^{23}$, et ce, tel qu'ont su l'exprimé Quinn et Degener dans l'introduction de leur étude. Évidemment, d'autres instruments internationaux viennent étayer la théorie selon laquelle les personnes handicapées, ou dites "vulnérables ", sont en droit de jouir des mêmes droits et des mêmes libertés que les autres citoyens. Nous avons sélectionné plusieurs conventions desquelles ressortent les principes déjà énoncés et s'en ajoutent d'autres, tels la participation politique et publique ainsi que le plein accès aux équipements de toute nature (équipements physiques, sociaux, économiques et culturels, mais aussi ceux relatifs à la santé, à l'éducation et à l'information). Nous avons également volontairement fait le choix de réduire notre analyse aux principes ci-dessus évoqués puisqu'ils nous semblent

21. Supra note 16 .

22. Pacte des droits économiques, sociaux et culturels, ibid., art. 16 : "Les États parties au présent Pacte s'engagent à présenter, conformément aux dispositions de la présente partie du Pacte, des rapports sur les mesures qu'ils auront adoptées et sur les progrès accomplis en vue d'assurer le respect des droits reconnus dans le Pacte ".

23. Supra note 13 à la p. 10. 
Personnes vulnérables et citoyenneté :

évolution et perspectives (2009-10) 40 R.D.U.S.

autour de l'accessibilité au processus de vote

fondamentaux relativement aux personnes visées et au sujet abordé.

Comme nous l'avons mentionné auparavant, l'Organisation des Nations Unies a adopté, en 1991, les Principes pour la protection des personnes atteintes de maladie mentale et pour l'amélioration des soins de santé mentale ${ }^{24}$ qui, comme le précisait Gendreau, créent "un cadre de référence à partir duquel les gouvernements pourront adapter leur législation conformément aux normes minimales énoncées pour la protection des patients des services de santé mentale "25. Ils s'appliquent aux personnes atteintes de maladie mentale, qu'elles soient ou non placées dans des établissements psychiatriques.

Il est fait mention, dès les premiers paragraphes du Principe 1, "qu'aucune discrimination fondée sur la maladie mentale n'est admise " et que "toute personne atteinte de maladie mentale a le droit d'exercer tous les droits civils, politiques, économiques, sociaux et culturels (...) ". Cette résolution comprend une énumération de vingt-cinq principes. Bien qu'essentielle, elle ne crée pourtant qu'un cadre formel servant de guide aux gouvernements et autres organismes de protection. Elle représente un standard minimum pour les personnes atteintes de maladie mentale. Et pourtant, les principes qui y sont énumérés sont difficiles à mettre en œuvre, selon les professionnels de la santé mentale qui y voient des "limitations et restrictions des droits des patients plutôt que l'expression d'une intention réelle de protection et [de] reconnaissance de [leurs] droits " 26 . En effet, dès lors qu'une intervention médicale s'avère nécessaire ou obligatoire, le principe de non-discrimination passe au second plan.

24. Supra note 8 .

25. Supra note 12 à la p. 508.

26. Voir Faculté de Médecine de l'Université de Genève, "Santé mentale et droits humains - Un compendium pédagogique " (19 août 2009), en ligne: Santé mentale et droits de l'homme <http://www. dhsantementale.net/cd/pat_intro.php>. 
Personnes vulnérables et citoyenneté :
(2009-10) 40 R.D.U.S.
autour de l'accessibilité au processus de vote

En juin 1999, une autre organisation, celle des États Américains, adopte la Convention interaméricaine sur l'élimination de toutes formes de discrimination envers les personnes handicapées ${ }^{27}$ qui affirme que ces dernières sont dotées des mêmes droits humains et jouissent des mêmes libertés fondamentales que les autres. Cette règle permet donc de réaffirmer le fait que les personnes handicapées peuvent s'intégrer pleinement dans la société sans être injustement exclues à cause de leur handicap. L'article 2 de la Convention énonce, en ce sens, que "[l]es objectifs de la présente Convention sont la prévention et l'élimination de toutes les formes de discrimination contre la personne handicapée et la création des conditions favorables à son insertion totale dans la société ${ }^{28}$. Elle propose également, explicitement, la coopération et la collaboration active des États parties afin de prévenir et d'éliminer la discrimination. Elle suggère le développement de moyens et de ressources destinés à faciliter l'insertion des personnes handicapées, dans des conditions d'égalité (article 4). Nous retrouvons donc dans cette convention, le principe d'égalité des droits et de son corollaire, celui de non-discrimination.

Quinn et Degener font du principe d'égalité une étude des plus intéressantes montrant qu'il faut "valoriser la différence " et accorder plus de "visibilité et jouissance de tous les droits fondamentaux dans l'égalité ". Ils rappellent que :

(...) tous les êtres humaines (sic) ont une valeur intrinsèque inestimable, mais qu'ils sont intrinsèquement égaux, quelles que soient leurs différences. (...) Cela ne veut pas dire qu'il n'existe pas de différences entre les individus. (...) cela signifie qu'une société hautement égalitaire est une société qui adopte une attitude tolérante et constructive à l'égard des différences humaines ${ }^{29}$.

27. Organisation des États Américains, Convention interaméricaine sur l'élimination de toutes formes de discrimination envers les personnes handicapées, 1999, en ligne : Commission interaméricaine des droits de l'homme <http://www.cidh.org/Basicos/French/o.handicapees.htm>.

28. Ibid., art. 2.

29. Supra note 13 à la p. 24. 
Personnes vulnérables et citoyenneté : évolution et perspectives (2009-10) 40 R.D.U.S. autour de l'accessibilité au processus de vote

Selon ces auteurs, le concept d'égalité s'explique selon trois interprétations: la première repose sur l'équité (c'est l'égalité formelle ou juridique) qui ne tient pas suffisamment compte des différences et qui, en bout de ligne, crée des droits illusoires; la seconde est celle qui postule "l'égalité des chances " selon laquelle "tout être humain a quelque chose à offrir à l'humanité et que les structures sociales devraient être conçues sur la base de l'intégration, avec pour principal objectif la responsabilisation de l'être humain "30; enfin, la troisième postule "l'égalité de résultats " grâce à laquelle chacun a droit à un minimum de droits peu importe son apport ou sa contribution à l'effort collectif. Par conséquent, le principe d'égalité vise à garantir la jouissance effective des droits fondamentaux de tout être humain indépendamment de ce qu'il reçoit ou offre lui-même à la société. Ce principe est un remède contre l'invisibilité dont pâtissent les personnes handicapées puisqu'il place tous les individus sur un même plan et leur reconnaît à tous un rôle actif dans la société.

Plus récemment, l'Organisation panaméricaine pour la Santé et l'Organisation mondiale de la Santé ont adopté une résolution intitulée Le handicap : prévention et réhabilitation dans le contexte du droit de la personne de jouir du niveau le plus élevé possible de santé physique et mentale et autres droits connexes ${ }^{31}$, en septembre 2006, dans laquelle elles prient les États membres :

d'envisager de ratifier ou d'approuver la Convention interaméricaine pour l'élimination de toutes les formes de discrimination des personnes handicapées [et les incitent] à sensibiliser l'ensemble du public aux questions de promotion et de protection des droits humains et des libertés fondamentales des personnes handicapées (art. 1 a).

30. Ibid. à la p. 26.

31. Organisation mondiale de la Santé et Organisation panaméricaine pour la Santé, Le handicap : prévention et réhabilitation dans le contexte du droit de la personne de jouir du niveau le plus élevé possible de santé physique et mentale et autres droits connexes, Rés. CD47.R1, Washington (D.C.), 2006, en ligne: Organisation panaméricaine pour la Santé <http://www.paho.org/French/GOV/CD/CD47.r1-f.pdf>. 
Personnes vulnérables et citoyenneté :
(2009-10) 40 R.D.U.S.
autour de l'accessibilité au processus de vote

Cette résolution est donc le pendant de la Convention interaméricaine mentionnée ci-dessus puisqu'elle cherche à rallier le plus grand nombre d'États et à sensibiliser les populations à la cause des handicapés.

Enfin, les deux conventions adoptées par l'Organisation des Nations Unies, respectivement en décembre 2006, la Convention relative aux droits des personnes handicapées ${ }^{32}$, et en mars 2007 , la Convention internationale pour la promotion et la protection des droits et de la dignité des personnes handicapées ${ }^{33}$, soulignent le fait que les droits des personnes handicapées font l'objet de plus en plus des préoccupations internationales. Ceci permet de diminuer peu à peu le caractère "invisible " dont souffrent ces personnes, mais aussi de développer des expertises favorisant la reconnaissance des droits en matière d'invalidité.

La première convention reprend l'ensemble des principes habituellement reconnus que sont la non-discrimination, la participation et l'intégrité pleines et actives à la société, l'égalité des chances, l'accessibilité, etc. Selon le site web des Nations Unies ONU Enable ${ }^{34}$ :

La Convention se veut un instrument des droits de l'homme comportant une dimension sociale explicite. Elle effectue un large classement des personnes handicapées par catégories et réaffirme que toutes les personnes qui souffrent d'une quelconque infirmité doivent bénéficier de tous les droits et libertés fondamentaux. Elle éclaire et précise la façon dont toutes les catégories de droits

32. Convention relative aux droits des personnes handicapées (19 août 2009), en ligne: Nations Unies <http://www.un.org/french/disabilities/ default.asp?id=1413>.

33. Convention internationale pour la promotion et la protection des droits et de la dignité des personnes handicapées, 2006, en ligne : Nations Unies <http://www.un.org/esa/socdev/enable/rights/convtextf.htm>.

34. Département de l'information (19 août 2009), en ligne : Secrétariat de la Convention relative aux droits des personnes handicapées du Département des affaires économiques et sociales des Nations Unies, en collaboration avec la Section du site Internet de l'ONU <http://www.un.org/french/disabilities/index.asp>. 
Personnes vulnérables et citoyenneté : évolution et perspectives (2009-10) 40 R.D.U.S. autour de l'accessibilité au processus de vote

s'appliquent aux handicapés et désigne les domaines où des adaptations permettraient à ces personnes d'exercer effectivement leurs droits, ainsi que les domaines où il y a eu violation de droits et où il convient de renforcer la protection de ces droits.

La seconde convention est un traité qui a été négocié pendant six ans pour être finalement approuvé par l'Assemblée générale des Nations Unies en mars 2007. Les organisations non gouvernementales actives dans le domaine du handicap y ont participé. La société civile s'est également impliquée dans son élaboration. Elle est entrée en vigueur en mai 2008 et a fait l'objet d'une consultation en ligne, sur sa ratification et son exécution, auprès d'organisations canadiennes, de personnes résidant au Canada et de Canadiens à l'étranger 35 . Cette Convention ne crée pas de nouveaux droits, elle consolide les droits existants. En fait, " elle est unique de par sa portée et son amplitude puisqu'elle recoupe tous les droits fondamentaux des personnes présentant un handicap "36. Dans le contexte qui nous intéresse plus particulièrement, remarquons qu'elle reconnaît le droit à la participation politique et à la vie publique (art. 29). Dans son préambule, elle " estim[e] que les personnes handicapées devraient avoir la possibilité de participer activement aux processus de prise de décisions concernant les politiques et programmes, en particulier, ceux qui les concernent directement "; elle " reconna[ît] qu'il importe que les personnes handicapées aient pleinement accès aux équipements physiques, sociaux, économiques et culturels, à la santé et à l'éducation ainsi qu'à l'information et à la communication pour jouir pleinement de tous les droits de l'homme et de toutes les libertés fondamentales ". Elle prévoit également, en son article $3 \mathrm{c}$ ) "la participation et l'intégration pleines et effectives à la société ".

35. Pour en savoir plus, voir Ressources humaines et Développement des compétences Canada, "Consultations publiques" (26 juillet 2010), en ligne : Ressources humaines et Développement des compétences Canada <http://www.hrsdc.gc.ca/fra/consultations_publiques/index.shtml> .

36. Supra note 4. 
Personnes vulnérables et citoyenneté :
(2009-10) 40 R.D.U.S.
autour de l'accessibilité au processus de vote

L'élaboration et l'application de ces normes internationales créent un corpus de règles qui tend à diminuer les inégalités entre les personnes "valides " et celles souffrant d'un handicap ou d'une incapacité entendue dans le sens des Nations Unies. Elles indiquent que la communauté internationale n'est pas indifférente au sort de ces personnes. La reconnaissance juridique de celles-ci n'a cependant pas toujours été aisée à mettre en œuvre.

\section{B) Les difficultés d'application}

C'est aux États qu'il appartient de veiller au respect des droits des personnes handicapées. Ils doivent réaliser les réformes nationales nécessaires au plein épanouissement des droits reconnus internationalement. Lorsqu'un État accepte de ratifier une convention internationale, il assume, en principe, les obligations qui en découlent. Il en dépend de la réputation de celui-ci auprès de la communauté internationale. Cela étant dit, comme le souligne Jocelyn Lecomte, les obligations internationales contractées, par exemple, par le Canada auprès des traités des Nations Unies dans le domaine des droits de la personne n'auront de valeur que si elles sont utilisées par les juristes au niveau national. L'étude de Gerard Quinn et Theresia Degener concernant les Nations Unies n'a, par ailleurs, pas manqué de souligner que :

[...] c'est aux États qu'il appartient au premier chef de veiller au respect des droits des personnes handicapées. C'est dire que la mise en œuvre et l'efficacité des mécanismes de l'ONU dans le domaine des droits de l'homme doivent être soutenues par des réformes nationales cohérentes. Les instruments relatifs aux droits de l'homme ne sont pas seulement une source d'inspiration, ils font obligation aux États de procéder à des réformes ${ }^{37}$.

Il est donc essentiel que les pays améliorent ou appliquent correctement leur législation en matière de santé mentale et de politiques d'aide aux personnes handicapées dans le respect des

37. Supra note 13 à la p. 11 . 
Personnes vulnérables et citoyenneté : évolution et perspectives (2009-10) 40 R.D.U.S. autour de l'accessibilité au processus de vote

droits fondamentaux des individus, car " les droits légaux sont des cadeaux vides (...) si ceux à qui ils sont accordés n'ont pas les moyens de les exercer "38. Les pays ont intérêt à respecter les instruments internationaux car, par le fait, ils assurent le respect, la protection et la promotion des droits ainsi définis.

Et pourtant, on remarque, selon l'Organisation mondiale de la Santé, qu'en 2005 de nombreux pays ne parviennent pas à mettre en place des politiques et des lois relatives à la santé mentale : $40 \%$ des pays ne possèdent pas de telles politiques et $64 \%$ n'ont pas de législation relative à la santé mentale ou en ont une qui date de plus de 10 ans $^{39}$. Une autre étude menée en 2004 sur la législation électorale de 63 pays démocratiques, énonce, quant à elle, que seuls quatre d'entre eux - le Canada, l'Irlande, l'Italie et la Suède - n'imposaient pas de restrictions, à l'échelon national, au droit de vote des personnes ayant un handicap mental ${ }^{40}$. En conséquence, sans mesure d'adaptation, ces personnes n'ont pas accès aux mêmes opportunités que les autres, elles sont isolées et marginalisées.

Au surplus, il est important de faire preuve de prudence et de vigilance face à la multiplication de normes toujours plus complexes et détaillées qui produirait une réaction inverse à l'effet voulu. Autrement dit, l'hypernormativité peut nuire parce qu'elle

38. Michael Mccubbin et David Cohen, "Les droits des usagers de services en santé mentale : Le nœud étroit du pouvoir, de la loi et de l'éthique " (1998) 23:2 Santé mentale au Québec 218.

39. Organisation mondiale de la Santé, Promouvoir et protéger les droits des personnes souffrant de troubles mentaux, note d'information no 1,7 décembre 2005, en ligne: Organisation mondiale de la Santé <http://www.who.int/mental-

health/policy/Promoting_and_protecting_rights_French.pdf $>$.

40. Louis Massicotte, André Blais et Antoine Yoshinaka, Establishing the rules of the Game : Elections Laws in Democracies, Toronto, University of Toronto Press, 2004 à la p. 27. Voir aussi l'analyse très documentée de Diane R. Davidson et Miriam Lapp, "L'évolution du droit de vote des Canadiens handicapés aux élections fédérales " (avril 2004), Perspectives électorales, en ligne: Élections Canada <http://www. elections.ca/eca/eim/article_search/article.asp?id=17\&lang=f\&frmPageS ize $=\&$ textonly=false $>$ [L'évolution du droit de vote des handicapés]. 
Personnes vulnérables et citoyenneté :
(2009-10) 40 R.D.U.S.
autour de l'accessibilité au processus de vote

offre des solutions à des problèmes qui sont de plus en plus éloignées des buts recherchés. L’affluence de réglementations crée aussi, comme certains l'ont déjà remarqué, "l'illusion d'une citoyenneté élargie enfin reconnue ", alors qu'en réalité, elle la fragmente ${ }^{41}$.

Quelle conclusion peut-on tirer de tout cela? Que seuls, les États n'ont peut-être pas les moyens de parvenir à résoudre les problèmes. En participant au mouvement des organisations non gouvernementales ou aux institutions nationales vouées à la défense des droits de l'homme, il n'y a pas de doute que les décideurs politiques sont sensibilisés à la reconnaissance des droits des personnes handicapées. La création de différentes commissions de défense des droits, à travers le monde, participe au changement des mentalités mais les procédures menées à l'intérieur de chaque État, qu'il s'agisse, par exemple, de réformes législatives, rendent parfois difficile l'application du principe d'égalité.

La mise en œuvre de ces différentes règles de droit international, par exemple au Canada, crée une difficulté qui tient au fait de l'application du principe du partage de compétences entre le provincial et le fédéral en vertu des articles 91 et suivants de la Loi constitutionnelle de $1867^{42}$. Une fois ratifié, un traité lie le Canada en droit international mais n'est pas contraignant en droit interne. Pour pouvoir produire ses effets en droit interne, une mesure législative doit être adoptée ou une décision des tribunaux canadiens doit être rendue. En principe, les traités internationaux

41. Supra note 12

42. Le partage de compétences est l'une des principales caractéristiques d'un régime fédératif. C'est le partage des pouvoirs entre le gouvernement central et les gouvernements des États membres. Les constituants canadiens ont confié les affaires d'importance nationale ou d'intérêt général au fédéral (Parlement central) et les affaires d'intérêt locales aux Provinces (Loi constitutionnelle de 1867 (R.-U.), 30\&31 Vict., c.3, reproduite dans L.R.C. 1985, app. II, $\mathrm{n}^{\circ} 5$ aux art. 91 et s.). Voir Réjean Pelletier, "Constitution et fédéralisme " dans Manon Tremblay, Réjean Pelletier et Marcel R. Pelletier, dir., Le parlementarisme canadien, 2e éd., Ste-Foy, Presses de l’Université Laval, 2000 à la p. 55. 
Personnes vulnérables et citoyenneté : évolution et perspectives (2009-10) 40 R.D.U.S. autour de l'accessibilité au processus de vote

portant sur les droits de la personne ratifiés par le Canada sont présentés à l'Assemblée nationale du Québec pour fins d'approbation, avant l'adhésion du Canada. Il n'en demeure pas moins que, pour la communauté internationale, c'est Ottawa, c'est-à-dire le fédéral, qui représente l'interlocuteur en matière de négociation et de mise en œuvre des traités. Ceci étant dit, le Canada a adopté des dispositions législatives garantissant le droit à l'égalité et l'obligation de prendre des mesures d'adaptation. Il s'agit, notamment, à l'échelon national, de la Charte canadienne, de la Loi canadienne sur les droits de la personne ${ }^{43}$ et de la Loi sur l'équité en matière d'emploi ${ }^{44}$. Dans le même sens, ont été mis sur pied le plan d'action intitulé Orientations futures du gouvernement du Canada concernant les personnes handicapées - la pleine citoyenneté : une responsabilité collective 45 et la Commission canadienne des droits de la personne. À l'échelon provincial, on retrouve différents organismes à but non lucratif. Au Québec, ont été créés l'Office des personnes handicapées du Québec ${ }^{46}$ et la Commission des droits de la personne et des droits de la jeunesse ${ }^{47}$. Enfin, à l'échelon international, le Canada est signataire de plusieurs conventions internationales. Avant d'en arriver à un tel stade, les organismes de défense des personnes handicapées ont dû mener combat. C'est donc, peu à peu,

43. Loi canadienne sur les droits de la personne, L.R.C. 1985, c. H-6, en ligne : Ministère de la Justice du Canada <http://lois.justice.gc.ca/fr/H$6 />$.

44. Loi sur l'équité en matière d'emploi, L.C. 1995, c. 44, en ligne : Ministère de la Justice du Canada <http://lois.justice.gc.ca/fr/E5.401/index.html>.

45. Canada, Ministère des Ressources humaines et Développement des compétences du gouvernement du Canada, Orientations futures $d u$ gouvernement du Canada concernant les personnes handicapées - la pleine citoyenneté : une responsabilité collective - janvier 1999, en ligne : Ressources humaines et Développement des Compétences Canada <http://www.rhdcc-hrsdc.gc.ca/fra/sm/ps/dsc/polsoc/publications / rapports/1999-000046/page00.shtml>.

46. Office des personnes handicapées du Québec, en ligne: <http://www.ophq.gouv.qc.ca/index.htm>.

47. Commission des droits de la personne et des droits de la jeunesse, en ligne : <http://www.cdpdj.qc.ca/> 
Personnes vulnérables et citoyenneté :
(2009-10) 40 R.D.U.S.
autour de l'accessibilité au processus de vote

franchissant une marche après l'autre, que le pays a reconnu les droits de ces personnes vulnérables.

\section{Le cadre national}

Il a fallu plus de vingt ans pour que les personnes handicapées et déficientes obtiennent la qualité d'électeurs au même titre que les autres citoyens canadiens. Un changement radical s'est opéré avec la décision de la Cour fédérale, en 1988, faisant des personnes handicapées de véritables sujets, titulaires de droits (A). À partir de là, toute une série de projets de réforme législative ont vu le jour, garantissant la jouissance de l'ensemble des droits de l'homme aux personnes handicapées sans pour autant empêcher une certaine stigmatisation à leur égard (B).

\section{A) Les changements apportés par la décision de la Cour fédérale du Canada}

En 1981, année internationale des personnes handicapées, un Comité spécial de la Chambre des communes concernant les invalides et les handicapés publie un rapport intitulé Obstacles. Il est alors recommandé au gouvernement canadien d'élaborer un système de vote postal, semblable à celui du Manitoba, et de former des préposés aux bureaux de scrutin capables de répondre aux besoins des électeurs handicapés; en bref, de rendre le processus de vote plus accessible. En 1982, la Charte canadienne appuie cet avis en offrant à tous, nonobstant une déficience mentale ou physique, les droits à l'égalité prévus dans la Constitution ${ }^{48}$. Elle énonce également, en son article 3, que " tout citoyen canadien a le droit de vote et est éligible aux élections législatives fédérales ou provinciales " et fait ainsi du droit de vote un droit fondamental. Le directeur général des élections au Canada incite le Parlement, à l'appui du rapport précité, à se pencher sur l'alinéa 14 (4) $f)^{49}$ de la Loi électorale de 1970 compte

48. Charte canadienne, supra note 5.

49. $\quad$ Loi électorale de 1970, supra note 3. 
Personnes vulnérables et citoyenneté : évolution et perspectives (2009-10) 40 R.D.U.S. autour de l'accessibilité au processus de vote

tenu de sa contestation possible fondée sur la Charte canadienne $e^{50}$.

Le rapport Obstacles est considéré comme le point de départ du débat sur les questions touchant les personnes handicapées au Canada. Il proposait d'élargir l'éventail de leurs droits. En effet, ceux-ci ne portaient plus seulement sur la santé et l'aide sociale, mais également sur les droits de la personne et la citoyenneté. En 1983, année qui marque le début de la décennie des Nations Unies pour les personnes handicapées, est nommé un ministre, au fédéral, responsable de la Condition des personnes handicapées. Le Bureau de la condition des personnes handicapées est également créé51. Le Comité permanent des droits de la personne et de la condition des personnes handicapées, est, quant à lui, créé en 1987. C'est donc en raison de ce contexte que l'accès égal, l'intégration économique et la participation concrète des personnes handicapées à la société canadienne sont encouragés.

C'est dans ce mouvement en faveur des droits des personnes handicapées et souffrant de déficience que la Cour fédérale du Canada a rendu sa décision. Dans la décision Conseil canadien des droits des personnes handicapées c. Canada, le 17 octobre $1988^{52}$, elle a déclaré inconstitutionnel le fameux article 14 (4) f) de La loi électorale de 1970 refusant le droit de vote à une personne "privée de la gestion de ses biens pour cause de maladie mentale ". Le juge Reed a estimé que cette disposition enfreignait l'article 3 de la Charte canadienne garantissant le droit de vote à tous les citoyens canadiens. Bien que l'article 1 de la Charte permette des restrictions - à la loi - dont la justification puisse se

50. Canada, Directeur général des élections du Canada, Rapport statutaire de $1984 d u$ directeur général des élections du Canada, Ottawa, Approvisionnements et Service Canada, 1984 à la p. 19.

51. Canada, Ministère des Ressources humaines et Développement des compétences du gouvernement du Canada, Bureau de la condition des personnes handicapées, en ligne: Ressources humaines et Développement des compétences Canada <http://www.hrsdc.gc.ca/fra/ condition_personnes_handicapees/mandat/index.shtml $>$.

52. Conseil canadien des droits des personnes handicapées c. Canada, [1988] 3 C.F. 622. 
Personnes vulnérables et citoyenneté :
(2009-10) 40 R.D.U.S.
autour de l'accessibilité au processus de vote

démontrer dans le cadre d'une société libre et démocratique ${ }^{53}$, la disposition visée ne respectait pas cette exigence et, dès lors, elle a été jugée " arbitraire ". En effet, comme le soulignait le juge " il est possible qu'une personne souffrant d'un handicap mental ou d'un trouble de la personnalité vive chez elle ou que ses biens soient assurés par sa propre famille. Or, cette personne qui ne serait visée par aucune des deux catégories mentionnées à l'alinéa 14 (4) $f)$ serait admissible à voter "54. En conséquence, l'argument avancé par la défense selon lequel "il est (...) erroné de présumer qu'une personne souffrant d'une incapacité mentale quelconque est incapable à tous points de vue, et notamment incapable de voter "55 a été retenu. D'ailleurs, "une personne incapable de prendre certaines décisions particulières peut être parfaitement capable d'en prendre d'autres „56. À l'appui de cet argument, le renvoi à l'ouvrage de Gerald B. Robertson, Mental Disability and the Law in Canada ${ }^{57}$ selon lequel "il est tout simplement erroné de présumer que les patients psychiatriques sont nécessairement incapables de voter. En effet, des recherches empiriques indiquent que le vote chez les patients psychiatriques se compare à celui du public en général "58. En conclusion, cette décision vient attester que le droit de vote ne peut être amputé aux personnes auxquelles il est fait référence.

53. Il s'agit de ce que les juristes appellent le test de Oakes à la suite de l'arrêt R. c. Oakes, [1986] 1 R.C.S. 103, rendu par la Cour suprême du Canada, interprétant l'article premier de la Charte canadienne et les critères de justification des limites aux droits constitutionnels. On peut lire en ce sens le récent ouvrage publié aux Éditions Thémis : Luc B. Tremblay et Grégoire Charles N. Webber, La limitation des droits de la Charte: Essais critiques sur l'arrêt R. c. Oakes / Critical Essays on R. $v$. Oakes, Montréal, Thémis, 2009. Parmi les limites raisonnables aux droits de l'individu, on retrouve, par exemple, l'interdiction de différents comportements répréhensibles comme les propos haineux (R. c. Keegstra, [1990] 3 R.C.S. 697) et l'obscénité (R. c. Butler, [1992] 1 R.C.S. 452).

54. Supra note 52.

55. Ibid.

56. Ibid.

57. Gerald B. Robertson, Mental Disability and the Law in Canada, 2e éd., Carswell, 1987.

58. Ibid. aux pp. 240-242. 
Personnes vulnérables et citoyenneté : évolution et perspectives (2009-10) 40 R.D.U.S. autour de l'accessibilité au processus de vote

Il s'agit, bien évidemment à l'époque, d'une grande victoire pour les groupes de défense des personnes handicapées, mais il faut attendre 1992 et 1993 pour que des changements concrets interviennent.

\section{B) Les projets de réforme législative et les perspectives d'avenir}

L'approche à la fois philanthropique et juridique à l'égard des personnes handicapées, entendu de manière globale, a contribué au développement de politiques de protection au profit de ces populations ainsi que des modifications concrètes à la loi.

En juin 1992, le projet de loi C-78 est adopté. Il accorde des changements législatifs majeurs garantissant l'accessibilité au processus de vote. Il incorpore à la Loi électorale du Canada plusieurs dispositions dont les suivantes: bureaux de vote itinérants dans les établissements où vivent des personnes âgées ou des personnes handicapées et programme d'information et d'éducation du public axé sur les personnes et les groupes de personnes susceptibles d'avoir des difficultés à exercer leur droit de vote.

Le Rapport Pleine participation 1992 permet également aux autorités provinciales et fédérales d'adopter le principe de la "vie autonome " et de présenter la vision, les principes et l'orientation stratégique devant permettre aux personnes handicapées de devenir membres, à part entière, de la collectivité et d'être davantage maîtres des décisions qui les concernent. Concrètement, des centres de vie autonome sont créés à travers tout le pays "pour fournir aux personnes handicapées de l'information, des services d'aiguillage, (...) un soutien à la défense des droits individuels et au développement des services "59.

59. Bulletin d'actualité du Gouvernement du Canada, division des affaires politiques et sociales, Les personnes handicapées : profil socio-économique et changements proposés par William R. Young, Canada, division des affaires politiques et sociales, 1997, en ligne : Gouvernement du Canada <http://dsp-psd.pwgsc.gc.ca/Collection-R/LoPBdP/CIR/954-f.htm>. 
Bien que des progrès significatifs soient apportés par le projet de loi C-78, il n'en demeure pas moins que certaines catégories d'individus ne peuvent accéder au vote. Il leur faudra encore attendre plusieurs semaines voire plusieurs années pour y parvenir. Le projet de loi C-114 réforme, de nouveau, la loi électorale, en mai 1993, et procure une accessibilité universelle au vote60. Il supprime les mesures qui privaient du droit de vote plusieurs groupes dont les personnes atteintes de déficience intellectuelle. La loi permet également à tous les électeurs de voter par bulletin spécial. Les personnes handicapées ou hospitalisées, les vacanciers ou les étudiants éloignés de leur domicile peuvent ainsi voter par la poste. Cette mesure vient remplacer le vote par procuration jugé trop complexe en raison notamment de la nécessité de présenter un certificat médical pour obtenir un certificat de procuration.

La situation des personnes handicapées a, de cette manière, largement contribué à l'adoption de changements à la loi. Les discussions avec les comités parlementaires chargés d'étudier les projets de loi (dont le Comité permanent des droits de la personne et de la condition des personnes handicapées) et avec le Conseil canadien des droits des personnes handicapées ont abouti à la rédaction d'un ensemble de propositions de réformes législatives détaillées ${ }^{61}$.

60. La Cour suprême du Canada accorde le droit de vote d'abord aux personnes condamnées à une peine de prison de moins de deux ans dans son arrêt Sauvé c. Canada (P.G.), [1993] 2 R.C.S. 438, puis à tous les détenus, peu importe la durée de leur peine : Sauvé c. Canada (Directeur général des élections), [2002] 3 R.C.S. 519. Quant aux sans-abris, ils obtiennent le droit de vote et de se présenter comme candidat à la suite des modifications de la Loi électorale, en 2000.

61. Conseil canadien des droits des personnes handicapées, Réforme législative pour les personnes handicapées - propositions de modifications, Winnipeg, Conseil canadien des droits des personnes handicapées, septembre 1991. Voir aussi les rapports rédigés par le Comité permanent des droits de la personne et de la condition des personnes handicapées tels que Comité permanent des droits de la personne et de la condition des personnes handicapées, S'entendre pour agir, Ottawa, juin 1990; Comité permanent des droits de la personne et de la condition des personnes handicapées, Les personnes handicapées, une réalité, Ottawa, 
Personnes vulnérables et citoyenneté : évolution et perspectives (2009-10) 40 R.D.U.S. autour de l'accessibilité au processus de vote

Malgré les progrès importants accomplis, la situation n'est pas totalement satisfaisante et il demeure nécessaire d'améliorer la législation concernant les droits des personnes souffrant de maladie mentale ou d'un handicap ${ }^{62}$. Gostin soulignait, en ce sens, qu' "il est temps de concevoir les problèmes auxquels doivent faire face les personnes atteintes d'incapacité mentale non seulement sous l'angle social mais aussi comme impératif des droits de l'homme "63. Il faut donc assurer une égalité et une participation entière des personnes considérées dans les sociétés démocratiques contemporaines. Les politiques et les programmes gouvernementaux doivent éliminer les obstacles et continuer de fournir des programmes spéciaux et des mesures spéciales d'aide conformes à cet objectif. Mais la simple existence d'une législation ne garantit pas, en elle-même, la non-violation des droits et en dépit de toute bonne volonté, il n'empêche qu'une question reste toujours en suspend : la maladie mentale et le handicap sont-ils des facteurs d'exclusion?

Il existe, aujourd'hui encore, une stigmatisation à l'égard des personnes souffrant de troubles mentaux qui limite leur accès à l'emploi, à l'éducation, au logement, à la citoyenneté. Elles sont,

mars 1993; Comité permanent des droits de la personne et de la condition des personnes handicapées, Des choix rentables pour tous, Ottawa, août 1993; supra note 59.

62. En 2007, " un demi-million de Canadiens avec des déficiences [devaient] (...) compter sur des programmes provinciaux d'aide sociale, ou des programmes analogues " selon le rapport Conseil des Canadiens avec déficiences, Le nouveau modèle opérationnel que tente d'instaurer la Commission canadienne des droits de la personne: les implications pour les personnes avec des déficiences par Yvonne Peters, Winnipeg, Conseil des Canadiens avec déficiences, 1 er novembre 2007, en ligne : Conseil des Canadiens avec déficiences <http://www.ccdonline.ca/fr/humanrights / promoting/new-business-model $>$.

63. Lawrence O. Gostin, "International human rights law and mental disability "The Hastings Center Report 34:2 (2004) 11 tel que cité dans Organisation mondiale de la Santé, Législation de la santé mentale. Conférence ministérielle européenne de l'OMS sur la santé mentale, Helsinki, 12-15 janvier 2005 : Relever les défis, trouver des solutions, en ligne: Organisation mondiale de la Santé <http://www.euro.who.int/ __data/assets/pdf_file/0008/98918/E88538.pdf>. 
Personnes vulnérables et citoyenneté :
(2009-10) 40 R.D.U.S.
autour de l'accessibilité au processus de vote

par voie de conséquence, limitées dans leur capacité de s’intégrer dans la société. Il s'agit là de ce que certains appellent la "violence structurelle " qui est une "forme pernicieuse et insidieuse de discrimination et d'abus, qui se traduit par une privatisation des droits "64. De sorte que les personnes vivant, notamment, avec des troubles mentaux ont rarement de voix politique, et ce, pour plusieurs raisons. D'une part, leurs propres difficultés mentales consument leur énergie et ébranlent toute intention de participer à l'arène politique. Comme le mentionnent Weisstub et ArboledaFlórez :

Les patients gravement malades sont préoccupés par leurs délires et leurs hallucinations; plusieurs sont trop paranoïaques pour simplement penser faire confiance à quelque forme que ce soit d'action de groupe, ils sont désorganisés à cause de leur comportement maniaque, ou trop déprimés pour simplement s'en préoccuper.

D'autre part, le sort de ces personnes ne fait pas débat en politique. Leur manque de visibilité restreint leurs chances de se faire entendre et les réglementations, quant à elles, sont axées bien plus souvent sur l'accès aux soins et aux traitements que sur la protection des droits fondamentaux. Leur participation insuffisante au débat démocratique donne lieu à l'élaboration de politiques qui les concernent directement mais auxquelles ils n'ont pas ou peu contribué. En conséquence, cela se traduit par un sentiment de rejet qui peut conduire à une désaffection vis-à-vis du processus démocratique et qui renforce certains stéréotypes.

Nous alléguons donc que la maladie mentale, au premier chef, mais aussi le handicap (physique ou intellectuel), dans une autre mesure, sont effectivement des facteurs d'exclusion, tant et si bien que les personnes qui en sont atteintes sont encore, parfois, considérées comme n'ayant pas la capacité de prendre de décisions dans leur propre intérêt, et par ricochet, encore moins

64. David Norman Weisstub et Julio Arboleda-Flórez, "Les droits en santé mentale au Canada : une perspective internationale " (2006) 31:1 Revue Santé mentale au Guébec 19. 
Personnes vulnérables et citoyenneté : évolution et perspectives (2009-10) 40 R.D.U.S. autour de l'accessibilité au processus de vote

dans l'intérêt collectif. Comme le soulignait Caroline Gendreau, "sans aucun doute, il est capital d'investir les différentes problématiques telles que l'âge, le handicap, la santé mentale et de prendre en considération les conditions matérielles nécessaires à la réalisation concrète des droits de la personne. Sans un tel investissement, ces droits demeureront inévitablement le privilège de quelques-uns malgré leur prétention à l'universalité (...) »65. Dans le même sens, l'État a une fonction primordiale qui tient au rétablissement des conditions rendant possible la citoyenneté. Cela signifie qu'au-delà de sa politique d'assistance, "l'État doit se montrer le régulateur ultime des intérêts existants dans la société (...) et aussi le dernier garant d'une conciliation qui part de la reconnaissance effective des droits fondamentaux pour tous les citoyens "66. Nonobstant, depuis l'adoption de la Charte canadienne, la loi électorale fédérale est l'une des plus souvent contestées au nom des droits et libertés dès lors que l'on met de côté le Code criminel. Ce qui tend à prouver que des aménagements à la loi sont encore nécessaires afin de coordonner droits fondamentaux et application légale.

\section{Conclusion}

Pour conclure, nous souhaitons souligner que plusieurs solutions tendant à faire évoluer cette situation ont déjà été mises en place. Le processus électoral au Canada est devenu progressivement plus accessible, notamment $\mathrm{du}$ fait de l'engagement des groupes de défense des personnes handicapées qui ont proposé et obtenu l'application d'un éventail de méthodes dans la lutte pour l'égalité électorale. Leur volonté d'action a finalement porté ses fruits et s'est concrétisée par l'application de mesures législatives et administratives au cours des trente dernières années. Concernant le vote, en particulier, des réformes significatives appliquées par Élections Canada ont permis aux handicapés de participer pleinement aux prises de décision. La

65. Supra note 12 à la p. 524

66. Supra note 1 à la p. 539. Yannuzzi discute de l'égalité et des droits entre " ceux qui se considèrent et ceux qui ne sont pas effectivement reconnus par l'État comme dignes d'être acceptés en tant que sujets de droits ". 
Personnes vulnérables et citoyenneté :
(2009-10) 40 R.D.U.S.
autour de l'accessibilité au processus de vote

fourniture d'une trousse d'information en braille et en gros caractère, la production d'une vidéo en langage des signes, l'utilisation du service téléphonique par téléscripteur, etc. en sont quelques exemples.

Le développement des nouvelles technologies d'information et de communication invite aussi à renouveler nos manières de voter. D'abord, Internet facilite l'accès aux informations et stimule la participation des citoyens en offrant la possibilité de débats et de forums virtuels. Ces forums sont des espaces de discussion, d'échange, entre groupes hétérogènes qui facilitent l'expression d'idées multiples. Ensuite, Internet est un moyen technique qui permet d'associer les citoyens à l'élaboration des politiques publiques. En effet, il est facile, de nos jours, de consulter les internautes et d'obtenir leur avis sur la mise en place d'une mesure spécifique, via un site Web. Enfin, le vote électronique apparaît comme le volet moderne d'une nouvelle forme d'expression. La perspective de voter via Internet obtenait, en 2004, un taux d'appui de $64 \%$ chez les groupes représentant les personnes handicapées, comparativement à $47 \%$ seulement chez le grand public 67 . Il s'agit là d'une voie à explorer bien qu'elle se heurte à de multiples difficultés dont celles de la sécurité absolue des réseaux, de la confidentialité du vote et de l'authentification des électeurs ${ }^{68}$.

Nous suggérons, enfin, plusieurs améliorations tendant à accroître la visibilité des personnes handicapées et par conséquent à leur reconnaître une véritable place dans la société : engager un dialogue continu avec les bénéficiaires afin qu'ils participent directement aux prises de décision qui les concernent; sensibiliser l'opinion aux droits des personnes handicapées et celles atteintes de maladie mentale afin d'éviter les rejets nés de l'ignorance; aider les organisations vouées à la défense de ces droits à faire le meilleur usage de la législation existante; faciliter les actions en justice à l'endroit des personnes handicapées; encourager la

67. L'évolution du droit de vote des handicapés, supra note 40.

68. Voir Denis Monière, Internet et la démocratie, Québec, Monière et Wollank Éditeurs, 2002 aux pp. 159 et ss. 
Personnes vulnérables et citoyenneté : évolution et perspectives (2009-10) 40 R.D.U.S. autour de l'accessibilité au processus de vote

désignation des personnes handicapées comme candidats aux élections (principalement dans les domaines qui les concernent); soutenir la recherche et développer l'expertise en matière de droit des personnes handicapées.

Afin de terminer sur une note optimiste, nous présumons qu'à l'appui des grands principes de droit international et sous la pression exercée par les organismes de promotion des droits de l'homme, les États auront la volonté d'améliorer la situation des personnes vulnérables vivant sur leur territoire. Depuis ces dernières années, des progrès ont été réalisés et il n'est pas illusoire d'espérer que la situation s'améliore plus encore, faisant des personnes handicapées et celles souffrant de maladie mentale des citoyens ordinaires. 